\title{
A Cartesian Ghost-Cell MultiGrid Poisson Solver for Incompressible Flows
}

\author{
Z.H. Ma, L. Qian, D.M. Causon, H.B. Gu, C.G. Mingham
}

\begin{abstract}
In this paper, a Cartesian ghost-cell multigrid Poisson solver is proposed for simulating incompressible fluid flows. The flow field is discretized efficiently on a rectangular mesh, in which the solid body is immersed. A small number of ghost mesh cells and their symmetric image cells are distributed in the vicinity of the solid boundary. Then Dirichlet and Neumann boundary conditions can be implemented with the aid of ghost and image cells. Chorin's projection method is used for the coupling of the velocity and pressure of the flows. Pointwise Gauss-Seidel iteration is implemented to solve the pressure Poisson equation. To speed up the convergence of the corresponding linear system, sub-level coarse meshes embedded with ghost and image cells are also introduced and operated in a V-cycle sequential. Several test cases including the classical ideal incompressible flow, the lid driven cavity flow and the viscous flows past a fixed/rotating cylinder are presented to demonstrate the accuracy and efficiency of the current approach.
\end{abstract}

\section{INTRODUCTION}

In the research field of computational physics, Cartesian grid method is an effective alternative to body-fitted structured and unstructured grid methods. The advantages of this method include the grid generation being a simple task, the superior property in implementing high order schemes as well as the lower computational storage requirement $[17,18]$. Furthermore, for the Cartesian grid cases with the mesh skewness or distortion can be eliminated.

A significant difference between a Cartesian grid and a body-fitted grid is that in the former the solid body is immersed in Cartesian grid cells [33], which means the grid lines (or volume surfaces) will not generally align with the solid boundaries. Unfortunately, this will cause difficulties in enforcing the boundary conditions directly on the grid lines (or volume surfaces). This problem has been addressed by many investigators, and great effort has been made to develop effective methods to overcome this deficiency.[21, 22, 23, 17, 18, 24].

Generally, the methods developed for the treatment of the solid boundary on a Cartesian grid can be divided into several categories including staircase approximation, immersed boundary, cut cell and ghost cell methods. 
Staircase approximation method makes use of staircase-like steps, whose edges are parallel to the grid lines of the regular grid, to handle the solid boundaries. As this method is rather rough, geometric errors will inevitably be introduced when dealing with curved boundaries [22, 25].

Immersed boundary method adds forcing terms to the governing equations, and the boundary is represented by a discrete set of body or surfaces forces to realize the boundary conditions. Its primary disadvantage is that the solution on the immersed boundary is smeared out, which means a sharp fluid-to-boundary interface cannot be resolved [24, 26].

Cut cell approach deals with the solid boundary as a sharp interface; bodies are cut out of a background Cartesian mesh and cells that are partially or completely cut are singled out for special treatment [22, 23]. By using this approach the smearing of boundary can be successfully prevented, but it might suffer significant stability and convergence problems when very small cut cells present near the solid body [33, 34]. Several methods such as "multi-valued dummy point" "the cell merging technique" and "rotated cells" are proposed to alleviate the time step limitation associated with small cut cells. However, these methods are rather complicated [17].

In order to avoid the stability problem caused by tiny-sized cut cells, a ghost cell method is proposed by Dadone et al. for compressible flows [33] in which only rectangular cells are used in the vicinity of a solid body. This method has since been successfully applied to two- and three-dimensional inviscid transonic flows [34, 32].

For solving viscous incompressible flows, the ghost cell method is also gaining popularity in deal with the solid boundary conditions. Ding et al. [30] adopted the ghost cell technique in a domain-free discretization method in which fractional step pressure correction method is taken to iteratively solve the velocity and pressure. Within the internal iterations, successive over relaxation (SOR) is used to solve the pressure Poisson equation to enforce the divergence-free condition for the velocity [30]. It is well known that finding the solution of the Poisson equation is the most time-comsuming part for this type of incompressible solver and a method with a quick convergence rate is essential for the overall efficiency of the method. In this aspect, a simple and efficient scheme like multigrid is highly desirable [29].

In this paper, a Cartesian mesh based ghost-cell multigrid Poisson solver (GCMGPS) is proposed for solving incompressible flows. The base Cartesian mesh is generated for the whole computational domain, and the solid body is immersed in this grid. Within the solid body, a set of mesh cells near the boundary is chosen and defined as ghost cells. Image cells which are symmetric to the ghost cells about the solid boundary are also introduced in the fluid flow region. Solid boundary conditions including Dirichlet and Neumann boundary conditions can then be implemented with the aid of ghost and image cells. Consequently, a simple five-point finite difference discretization scheme can be applied in the whole computaional domain. Chorin's projection method is adopted for the coupled solution of the velocity and pressure of incompressible flows. Several levels of coarse meshes embedded with ghost and image cells are 
introduced to speed up the convergence rate of the pressure Poisson equation.

This paper is organized as follows. In Section 2, Cartesian ghost cell multigrid Poisson solver is described in detail. Then, Chorin's projection method for viscous incompressible flows is briefly discussed in Section 3. Numerical examples including ideal incompressible flow, lid driven cavity and viscous flows past a fixed/rotating cylinder are presented in Section 4 to validate the proposed method. Some conclusions are drawn in the last section.

\section{GHOST-CELL MULTIGRID METHOD}

In two-dimensional Cartesian coordinate system, the Poisson equation can be written as

$$
\frac{\partial^{2} \phi}{\partial x^{2}}+\frac{\partial^{2} \phi}{\partial y^{2}}=f, \phi \in \Omega
$$

in which $\phi$ may represent the fluid flow pressure, velocity potential or other physical variables, $f$ is a source function and $\Omega$ is the computational domain. On the boundary $\partial \Omega, \phi$ usually needs to satisfy the Dirichlet boundary condition

$$
\phi=\sigma
$$

where $\sigma$ is a prescribed function value, or Neumann boundary condition

$$
\frac{\partial \phi}{\partial n}=0
$$

\subsection{Ghost-cell boundary condition}

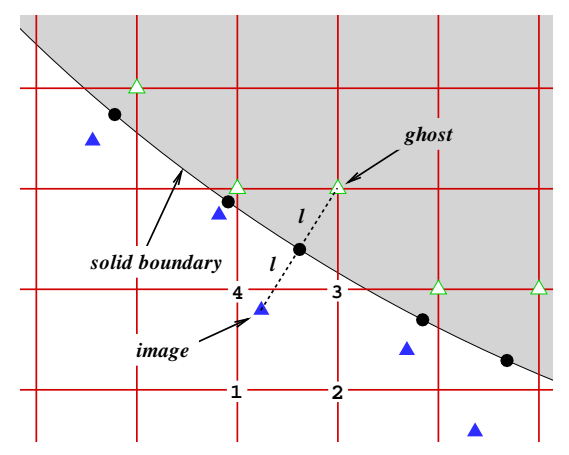

Figure 1: Ghost-cell treatment. (Black circle $\bullet$ represents the solid boundary point.)

For arbitrary configurations, solid curved boundaries are usually not aligned with Cartesian grid lines and therefore boundary conditions cannot be directly implemented on them. This brings a drawback to the Cartesian grid based method compared to the body-fitted meshes in which boundary conditions can 
satisfied in a straightforward way. In order to overcome this deficiency and to avoid the complexity in other methods such as the cut cell approach, a ghost cell method $[25,32,29,33,34]$ is employed in the present work.

Figure 1 illustrates the essential idea of the ghost cell method for curved boundaries. The base Cartesian grid is generated to cover the whole domain, and the solid body is immersed in the rectangular cells. Before the start of the numerical computation, all mesh points need to be classified into different categories according to their relative positions to the solid boundary. All the mesh points in the fluid region are defined as fluid points with mark $=1$ while all the mesh points inside a solid body are defined as solid points with mark $=0$. Within the second group, the mesh points belong to the cells which are patially cut by the background Cartesian grid lines are defined as ghost cells (or ghost points). In Figure 1, the open triangles represent the ghost points, the solid triangles are their symmetric image points about the solid boundary. The black dots are on the boundary and are at the centers between ghost and image points. The distance from a ghost or image point to the solid boundary is indicated by $l$.

For clearness, we use subscripts $b, g$ and $i$ to represent the solid boundary, ghost and image points, respectively. Then the physical boundary condition for $\phi_{b}$ can be transformed to the requirement of $\phi_{g}$ and $\phi_{i}$. For simplicity, $\phi_{b}$ can be treated as the average of $\phi_{g}$ and $\phi_{i}$

$$
\phi_{b}=\frac{1}{2}\left(\phi_{g}+\phi_{i}\right)
$$

and the normal derivative of $\phi$ at the boundary can be calculated by

$$
\frac{\partial \phi_{b}}{\partial n}=\frac{\phi_{i}-\phi_{g}}{2 l}
$$

Using Taylor series analysis, it is not difficult to know that Eq. (4) and (5) have the second order accuracy in term of $l$. For the Dirichlet boundary condition $\phi_{g}$ can then be computed by

$$
\phi_{g}=2 \phi_{b}-\phi_{i}
$$

and for Neumann boundary condition it can calculated by

$$
\phi_{g}=\phi_{i}-2 l \frac{\partial \phi_{b}}{\partial n}
$$

The value of the image point $\phi_{i}$ can be obtained by a bilinear interpolation

$$
\phi_{i}=k_{1} \phi_{1}+k_{2} \phi_{2}+k_{3} \phi_{3}+k_{4} \phi_{4}
$$

where $k_{1}, k_{2}, k_{3}$ and $k_{4}$ are the interpolation coefficients, and they are given by

$$
\left\{\begin{aligned}
k_{1} & =\left(x_{2}-x_{i}\right)\left(y_{3}-y_{i}\right) / S_{1234} \\
k_{2} & =\left(x_{i}-x_{1}\right)\left(y_{3}-y_{i}\right) / S_{1234} \\
k_{3} & =\left(x_{i}-x_{1}\right)\left(y_{i}-y_{1}\right) / S_{1234} \\
k_{4} & =\left(x_{2}-x_{i}\right)\left(y_{i}-y_{1}\right) / S_{1234}
\end{aligned}\right.
$$

where $S_{1234}$ is the area of the cell in which the image point lies. 


\subsection{Multigrid Poisson solver}

When simulating incompressible flows by fractional-step projection methods, the solution of the pressure Poisson equation is needed to maintain the divergencefree of the velocity field[1, 2]. As this is normally the most time-consuming part of the computation it is crucial that an efficient scheme like multigrid method is utilised [29].

Standard iterative methods including Jacobi, Gauss-Seidel (GS) and successive over-relaxation (SOR) are simple and easy to apply. In the context of ghost cell methods, some researchers have used these techniques to solve the resultant linear algebraic equations [30]. However, this type of methods has a tendency of slow convergence which becomes more prominent when the mesh is refined [35]. It triggers off the necessity of applying multigrid method(MG) to speed up the convergence. MG has been proved to be effective in terms of solving problems with $N$ unknowns with $O(N)$ work and storage [38, 39] and has been adopted in this study.

The basic theory of MG is to eliminate the low-frequency and high-frequency parts of numerical errors at the same pace. On the fine mesh, high-frequency errors are removed rapidly, but the low-frequency part is rather stiff which reduces slowly even after many iterations. MG successively adopts several levels of coarse meshes to further expunge the low frequencies. The solution on the coarse mesh is transferred back to improve the solution on the fine mesh. Usually, these two steps are called restriction and prolongation (or correction) [35]. As more than two levels of grid are used in the present work, we apply a classical "V-cycle" to recursively solve linear system from the finest mesh to the coarsest mesh then from the coarsest to the finest. A brief description of MG is given as follows.

For simplicity, Eq. (1) is written into a simplified form

$$
\mathcal{L} \phi=f
$$

where $\mathcal{L}$ is the Laplace operator. Using subscript $h$ to indicate the fine mesh, then the solution to the linear system on the fine mesh can be expressed by

$$
\phi_{h}=\mathcal{L}_{h}^{-1} f_{h}
$$

Supposing $\tilde{\phi}_{h}$ is the approximate solution and $e_{h}$ is the error, the residual of Eq. (10) on the fine mesh can be defined as

$$
r_{h}=f_{h}-\mathcal{L}_{h} \tilde{\phi}_{h}=f_{h}-\mathcal{L}_{h}\left(\phi_{h}-e_{h}\right)
$$

Then it is restricted to the coarse mesh denoted by subscript $H$

$$
r_{H}=\mathcal{I}_{h}^{H} r_{h}
$$

On the coarse mesh, the following equation is required to be accurately solved

$$
\mathcal{L}_{H} e_{H}=r_{H}
$$


The error on the fine mesh is then approximated by an extrapolation

$$
e_{h}=\mathcal{I}_{H}^{h} e_{H}
$$

Consequently, the approximate solution on the fine mesh can be improved by

$$
\phi_{h}^{*}=\tilde{\phi}_{h}+e_{h}
$$

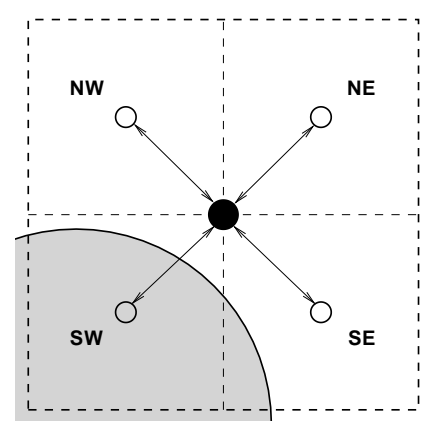

Figure 2: Restriction and prolongation between fine and coarse meshes. (The open circles are fine mesh cell centers, the solid symbol is a coarse mesh cell center.)

For Poisson equations, $\mathcal{I}_{h}^{H}$ and $\mathcal{I}_{H}^{h}$ are two linear extrapolation functions designed for restriction and prolongation, respectively. If the physical variable $\phi$ is stored at the mesh cell center, then the restriction function can be constructed by a volume (or area) average

$$
r_{H}=\frac{r_{h, N W} \cdot S_{h, N W}+r_{h, N E} \cdot S_{h, N E}+r_{h, S W} \cdot S_{h, S W}+r_{h, S E} \cdot S_{h, S E}}{S_{h, N W}+S_{h, N E}+S_{h, S W}+S_{h, S E}}
$$

and the prolongation is even more simple

$$
e_{h}=e_{H}
$$

These procedures are clearly shown in Figure 2. The open circles represent the fine mesh cell centers, and the subscripts NW, NE, SW and SE are used to denote these centers. The solid dot that is slightly bigger than the open circles displays a cell center on the coarse mesh. A segment of the boundary is depicted by the solid curve, and the gray part surrounded by this curve is a portion of the solid body. For conciseness, only a general explanation of MG is presented in this paper, and more details of the method can be found in the book of Hackbush[35].

For simple configurations that align with Cartesian grid, MG is easy to realize because the coarse mesh can be well handled by blanking the cells located in the solid body [12]. However for curved boundaries care should be exercised as as simple blanking will violate boundary conditions. In this context, ghost 

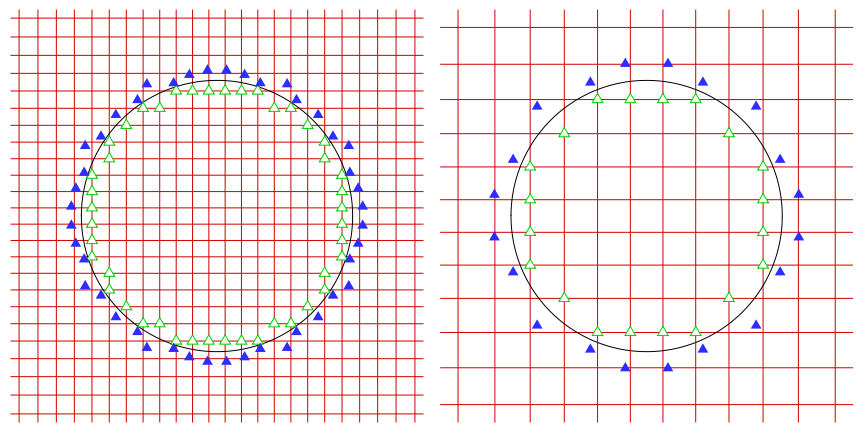

Figure 3: Ghost and image cells on fine and coarse meshes. (Open triangles are ghost cells and solid triangles are image cells)

cells and their images are also required on coarse meshes and this has been clearly shown in Figure 3. The left figure exhibits the ghost and image cells on a fine mesh, and the right figure displays the ones on a coarse mesh. In this figure, the open triangles are ghost cells, and their images are represented by solid triangles. On the coarse mesh, the boundary condition is implemented by using the same method described in section 2.1. If there are several levels of coarse meshes then the corresponding ghost and image cells are needed for each level to satisfy the boundary conditions.

\section{PROJECTION METHOD}

The advective form of incompressible Navier-Stoke equations are given by

$$
\left\{\begin{aligned}
\nabla \cdot \vec{V} & =0 \\
\frac{\partial \vec{V}}{\partial t}+\vec{V} \cdot \nabla \vec{V} & =-\nabla p+\frac{1}{R e} \nabla^{2} \vec{V}
\end{aligned}\right.
$$

where $\vec{V}$ is the velocity vector, $p$ is the pressure and $R e$ represents the Reynolds number defined as

$$
R e=\frac{\rho U D}{\mu}
$$

in which $\rho$ is density, $U$ is the free-stream velocity, $D$ is the characteristic length or diameter of the solid body and $\mu$ is the dynamic viscosity.

As the density of the incompressible fluid flow is a constant, its time derivative in the continuity equation vanishes. Consequently, the corresponding governing equations are not hyperbolic and straightforward time-marching methods designed for compressible flows are not suitable any more. For this reason, Chorin's projection method $[1,2]$ is applied in the present work. A brief description of this method is given as follows.

Firstly, an intermediate velocity vector field $\vec{V}^{*}$ is calculated by ignoring the 
pressure gradient term in the momentum equation

$$
\frac{\vec{V}^{*}-\vec{V}^{n}}{\Delta t}=-\left(\vec{V}^{n} \cdot \nabla\right) \vec{V}^{n}+\frac{1}{R e} \nabla^{2} \vec{V}^{n}
$$

where $\vec{V}^{n}$ is the velocity at the $n$th time level. In the next step, the velocity at the new time step will be computed by taking the pressure gradient into account

$$
\frac{\vec{V}^{n+1}-\vec{V}^{*}}{\Delta t}=-\nabla p^{n+1}
$$

Rewriting the above equation for the velocity at $(n+1)$ th time level, we have

$$
\vec{V}^{n+1}=\vec{V}^{*}-\Delta t \nabla p^{n+1}
$$

Computing the right-hand side of the above equation requires a knowledge of the pressure at the $(n+1)$ th time step. Taking the divergence of equation (23), then we have the pressure Poisson equation

$$
\nabla^{2} p^{n+1}=\frac{1}{\Delta t}\left(\nabla \cdot \vec{V}^{*}-\nabla \cdot \vec{V}^{n+1}\right)
$$

The velocity at this time step is required to satisfy the divergence-free (or solenoidal) condition

$$
\nabla \cdot \vec{V}^{n+1}=0
$$

Therefore, equation (24) is simplified as

$$
\nabla^{2} p^{n+1}=\frac{1}{\Delta t} \nabla \cdot \vec{V}^{*}
$$

This equation is solved by the Cartesian ghost-cell Multigrid method described in section 2. Both the advective and viscous terms in Eq. (21) are discretized by central difference scheme.

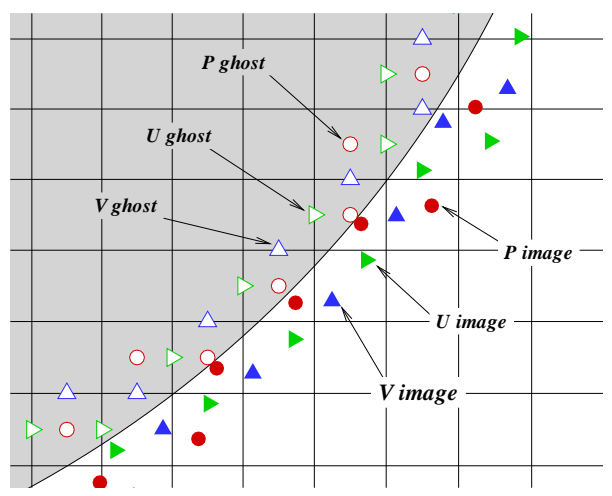

Figure 4: Boundary treatment on staggered grid. 
On the solid body, Neumann boundary condition for pressure is implemented, and no-slip condition for the velocity is also required

$$
\vec{V}=\vec{V}_{b}=u_{b} \vec{i}+v_{b} \vec{j}
$$

In order to implement the no-slip condition, ghost cells for $u$ and $v$ components are also needed. For a collocated grid where the information of $u$ and $v$ are stored at the same place as $p$, only one group of ghost cells are needed. However, a collocated grid arrangement cannot guarantee strong coupling between the velocities and the pressure. To suppress possible oscillations in pressure and velocity fields [37], a fully staggered grid is used in the present work. Therefore, we need to introduce ghost and image cells for $u, v$ and $p$ separately as shown in Figure 4. The pressure ghost cells and their image cells are depicted by open circles and bullets respectively. For the velocity component $u$, the symbol $\triangleright$ and represent the ghost and image cells. For $v$, the ghost and image cells are displayed by $\triangle$ and $\boldsymbol{\Delta}$. Having defined the ghost and image cells for the velocity, its components at ghost cells are given by

$$
\left\{\begin{array}{c}
u_{g}=2 u_{b}-u_{i} \\
v_{g}=2 v_{b}-v_{i}
\end{array}\right.
$$

where $u_{i}$ and $v_{i}$ are obtained by the interpolation scheme described by formula (8).

\section{NUMERICAL RESULTS}

In order to validate the proposed GCMGPS approach, in this paper the ideal incompressible flow over a cylinder governed by the Laplace equation is firstly chosen as a benchmark test. Then the method is extended for the solution of the incompressible Navier-Stokes equation with the test cases including the lid driven cavity flow and flows over a fixed/rotating circular cylinder at low Reynolds number.

\subsection{Ideal incompressible flow}

For Eq. (1), supposing that $\phi$ is the velocity potential and $f$ is zero, we have the Laplace equation which can be used to describe the incompressible irrotational flow around a circular cylinder. The exact solution of $\phi$ for this flow problem is given by

$$
\phi(r, \theta)=U\left(r+\frac{a^{2}}{r}\right) \cos \theta
$$

where $U$ is the speed of free stream, $a$ is the radius of the cylinder [40].

In the present work, the computaional domain chosen for this problem is a square with side length of 6 , the radius of the cylinder is $a=0.5$, the speed of free stream is $U=1$. On the farfield boundaries of the domain, $\phi$ is prescribed according to Eq. (29). The multigrid ghost cell method has been used to satisfy 
the Neumann boundary condition at the solid surface. Five levels of uniform meshes are utilized to cover the flow field. The number of the mesh points on the finest grid is $256 \times 256$, and the number of corresponding ghost cells around the cylinder is 120 . For the coarsest level, the number of mesh points is $16 \times 16$, and the number of ghost cells is 4 . In the present work, we apply GS as the basic smooth iterator.
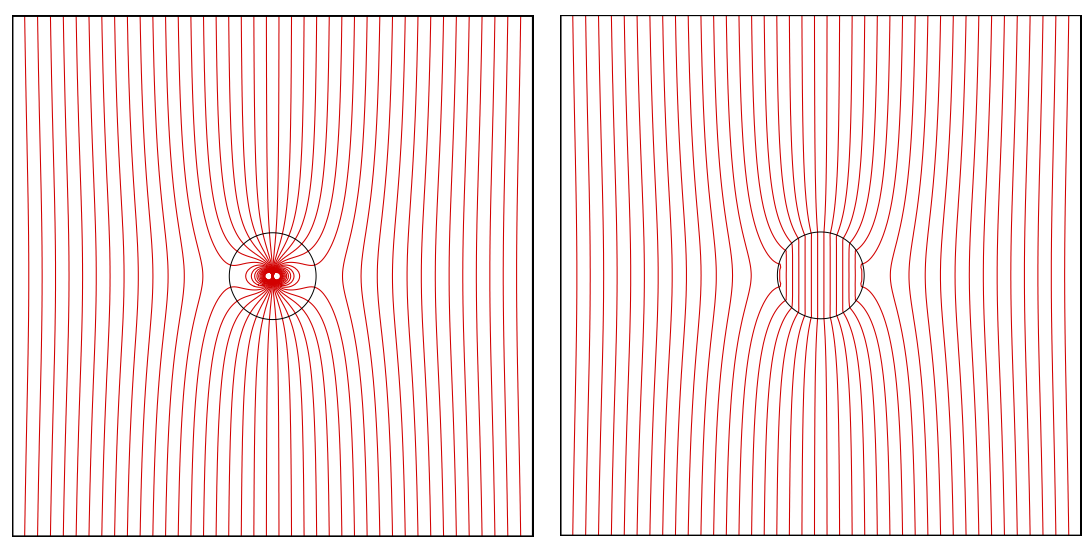

Figure 5: Velocity potential contours in the flow field. (Left: exact, Right: numerical)

Figure 5 shows the velocity potential contours. The left figure is for the exact solution and the right figure represents the numerical result. The range of the velocity potential values for the plotted contours is from -2.9 to 2.9. It is clear from this figure that the flow pattern from the numerical resutls is nearly the same as that of the exact solution. In order to examine the accuracy of the method quantitively, a comparison of $\phi$ between numerical result and exact solution is given in Figure 6 and 7. Figure 6 shows $\phi$ around the surface of the cylinder. Figure 7 presents the value of $\phi$ along the horizontal line across the cylinder center. The region from $x=-0.5$ to $x=0.5$ is located in the solid body. Obviously, a good agreement with the exact solution is achieved in the fluid flow region.

The convergence histories for both the GS and the GCMGPS methods are shown in Figure 8 whewe the residual defined by $L_{2}$ norm of the errors has been used as the indicator. It can be seen from the figure that the convergence rate of the Poisson solver with ghost-cell multilevel grids is much faster than the single level GS solver. For the GS method, after initial several dozens of iterations, the convergence rate will slow down substantially while for the GCMGPS method, it can maintain a nearly constant rate convergence throughout the computation. 


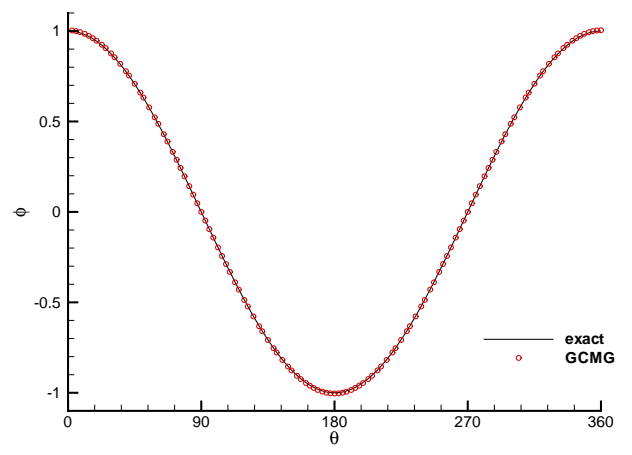

Figure 6: The value of $\phi$ on the cylinder.

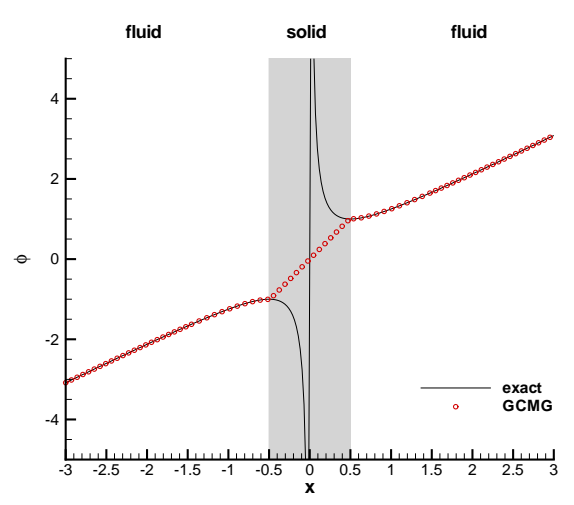

Figure 7: The value of $\phi$ along the horizontal line across the cylinder center. 


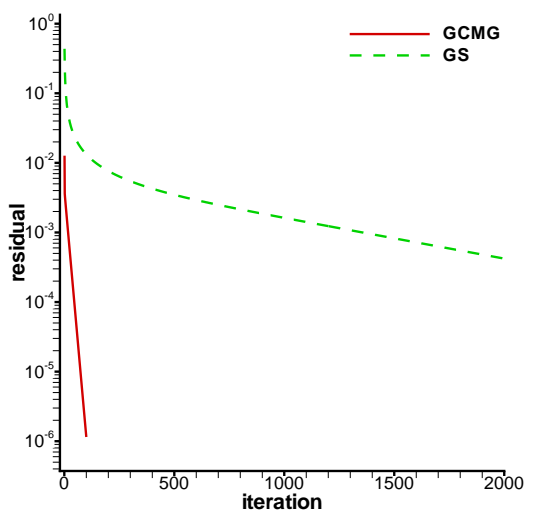

Figure 8: Convergence history of residual for ideal incompressible flow.

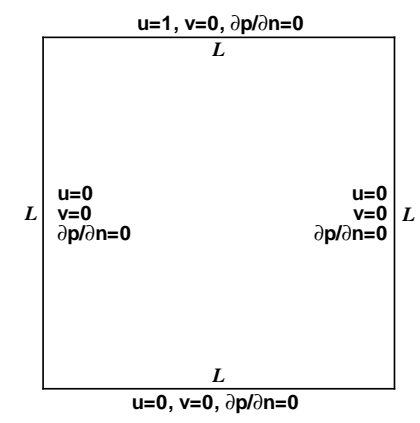

Figure 9: Geometry and boundary conditions of lid driven cavity $(L=1)$. 


\subsection{Lid driven cavity}

The lid driven cavity flow, due to its simplicity in geometry, is a popular test case for many researchers in CFD to validate their new schemes. The computational domain is a square box with a side length of one unit as shown in Figure 9. The top boundary of the domain is moving along $x$ direction with a constant speed $u=1$ and the other three boundaries are fixed,

The Reynolds number chosen for this work is $R e=5,000$. A uniform mesh with $128 \times 128$ points is used to discretize the flow field. To speed up the convergence of pressure Poisson equation, six levels of coarsening meshes are incorporated into the multigrid V-cycle process. For this test case as all the boundaries of the cavity align with the grid lines, the implementation of the boundary conditions using the ghost cell method is straightforward. Despite this, it can still be used to test the accuracy and effectiveness of the multigrid Poisson solver when combined with the projection method.

The calculated flow pattern in terms of streamlines is shown on the left of Figure 10 from which it can be seen that at the chosen Reynolds number vortices with different sizes are present in the flow field. A close-up view of the two tiny secondary vortices on either side of the two bottom corners is shown in Figure 11, which demonstrates the capability of the current approach in capturing the small flow structures. On the right of Figure 10, the velocity profiles for its $u$ component along the vertical centre line $(x=0.5)$ and its $v$ component along the horizontal centre line $(y=0.5)$ are compared between the current numerical result (solid line) and that of Ghia (depicted by o) [13] showing a very good agreement.
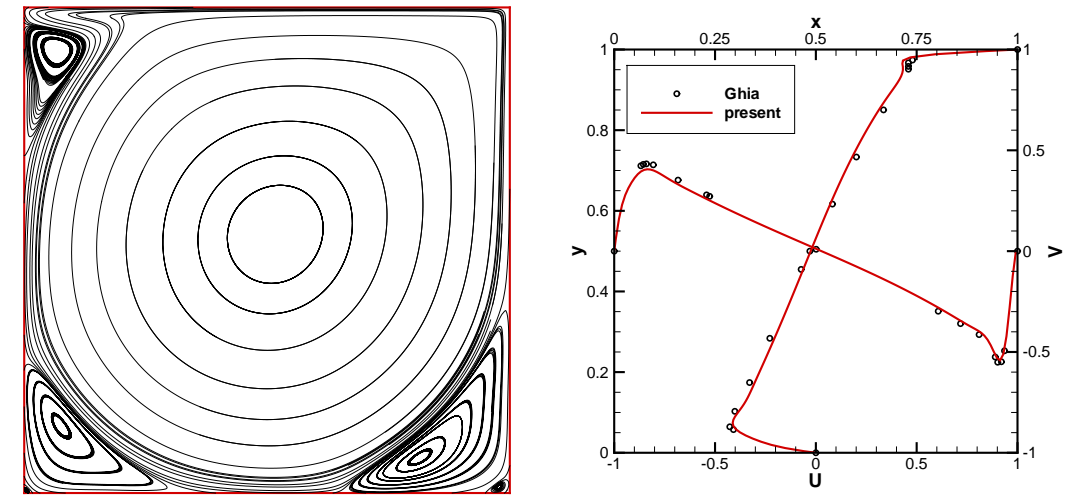

Figure 10: Lid driven cavity. Left: streamline; Right: center-line velocity profiles of $u$ and $v(R e=5000)$. 

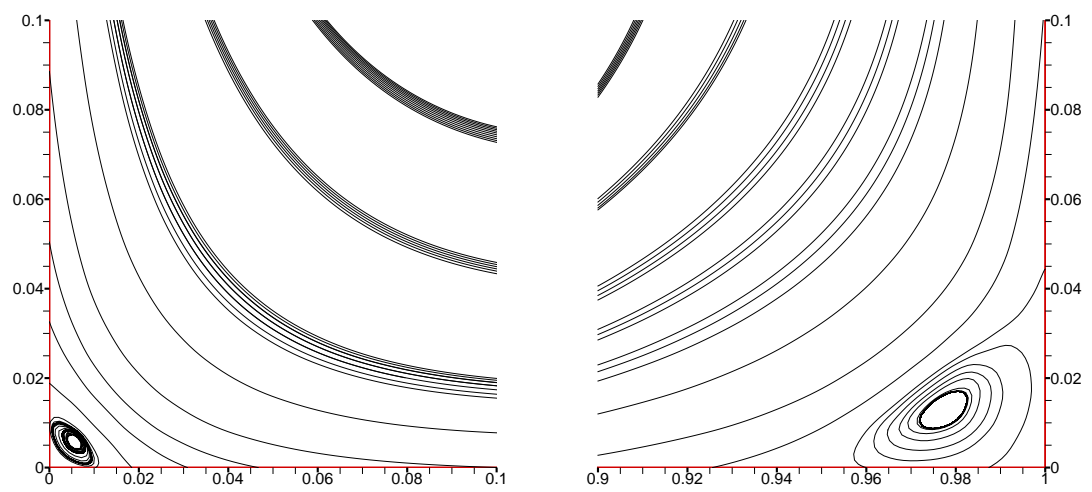

Figure 11: Close view of streamline pattern in the cavity $(R e=5000)$. Left: bottom-left corner; Right: bottom-right corner.

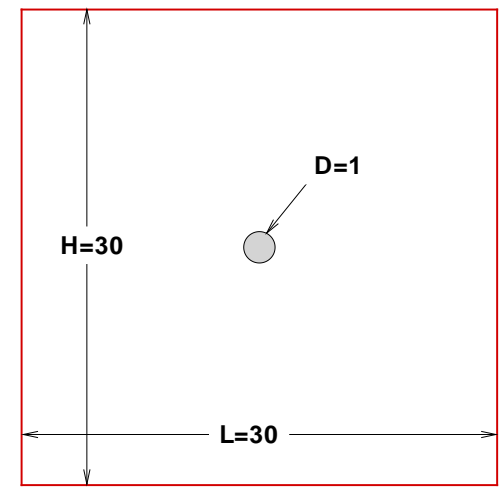

Figure 12: Geometry for viscous flows past a circular cylinder. 


\subsection{Flow past a fixed cylinder}

To test the capability of the present method to deal with flow problems with curved boundaries, the incompressible viscous flows around a fixed circular cylinder at the selected low Reynolds numbers of 20,40 and 100 are then calculated.

Figure 12 shows the geometries of the computational domain and the circular cylinder. The diameter of the cylinder is $D=1$ and the length and the height of the square-shaped domain are both 30. At the left boundary, the free stream inflow condition is specified with a constant velocity of $u=1$, and at the right boundary an outflow boundary is implemented. The top and bottom boundaries are impermeable [24]. A non-uniform mesh with $256 \times 256$ points is utilized to cover the flow field. The cells with a minimum size of $\Delta x=\Delta y=0.03$ are located around the surface the cylinder. To implement the solid boundary condition, 94 pressure ghost cells, $92 u$ ghost cells and $92 v$ ghost cells are identified inside the solid body. The same number of corresponding image cells are distributed around the cylinder for this level of the mesh. In order to speed up the convergence of pressure Poisson equation, four levels of coarser meshes are also introduced. On the coarsest mesh, the number of ghost and image cells are both 4 .

For the Reynolds number of 20 and 40, the streamline patterns are shown in Figure 13 in which the two symmetric vortices behind the cylinder can be clearly seen for each Reynolds number and also as expected it can be found that the wake length $L_{w}$ increases with Reynolds number. Figure 14 displays the pressure contours in the flow filed showing a smooth pressure fields. To indicate the accuracy of the current approach, the hydrodynamic parameters calculated including the drag coefficient and the length of wake are compared with the results from other researchers as listed in Table 1 from which it can be seen that our results agree reasonably well with the cited work [14, 26, 3, 30, 24].

Table 1: Hydrodynamic parameters of flows past a fixed cylinder

\begin{tabular}{l|llllll}
\hline Contribution & \multicolumn{3}{|c}{$R e=20$} & \multicolumn{2}{c}{$R e=40$} & \multicolumn{2}{c}{$R e=100$} \\
& $C_{D}$ & $L_{w}$ & $C_{D}$ & $L_{w}$ & $\bar{C}_{D}$ & $S_{t}$ \\
\hline Tritton [14] & 2.08 & - & 1.59 & - & 1.267 & 0.164 \\
Hartmann [26] & 2.043 & 0.972 & 1.535 & 2.240 & 1.358 & 0.164 \\
Fornberg [3] & 2.00 & 0.91 & 1.50 & 2.27 & - & - \\
Ding [30] & 2.14 & 0.94 & 1.58 & 2.32 & 1.391 & 0.166 \\
Chung [24] & 2.05 & 0.96 & 1.54 & 2.30 & 1.392 & 0.172 \\
Zhang [7] & - & - & - & - & 1.425 & 0.173 \\
Present & 2.08 & 0.92 & 1.56 & 2.32 & 1.410 & 0.167 \\
\hline
\end{tabular}

For the case of $R e=100$, the flow is not stable any more and any perturbation in the flow will cause asymmetry in the wake region which will eventually trigger the process of vortex shedding. The computed mean drag from the current method is $\bar{C}_{D}=1.410$ which is close to other results, see Table 1 . The 

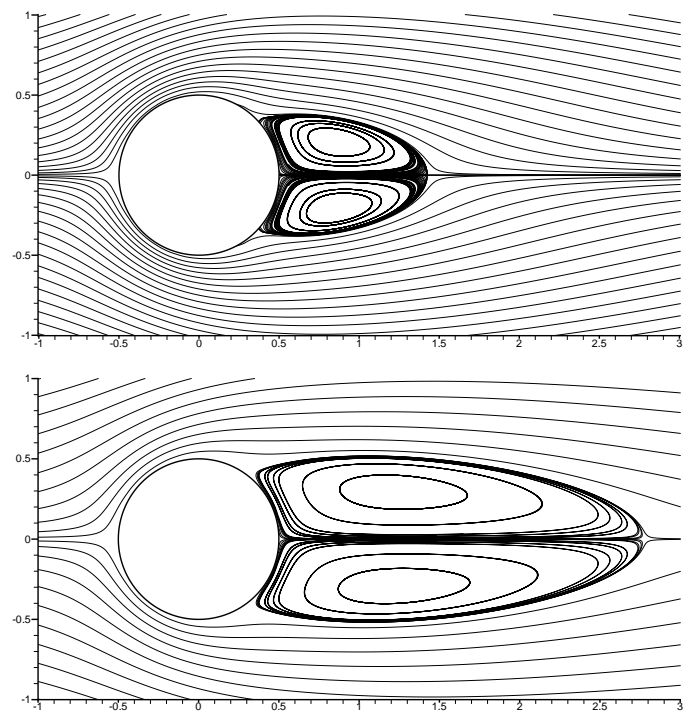

Figure 13: Streamline pattern of steady flows over a fixed cylinder (Top: $R e=$ 20; Bottom: $R e=40)$.
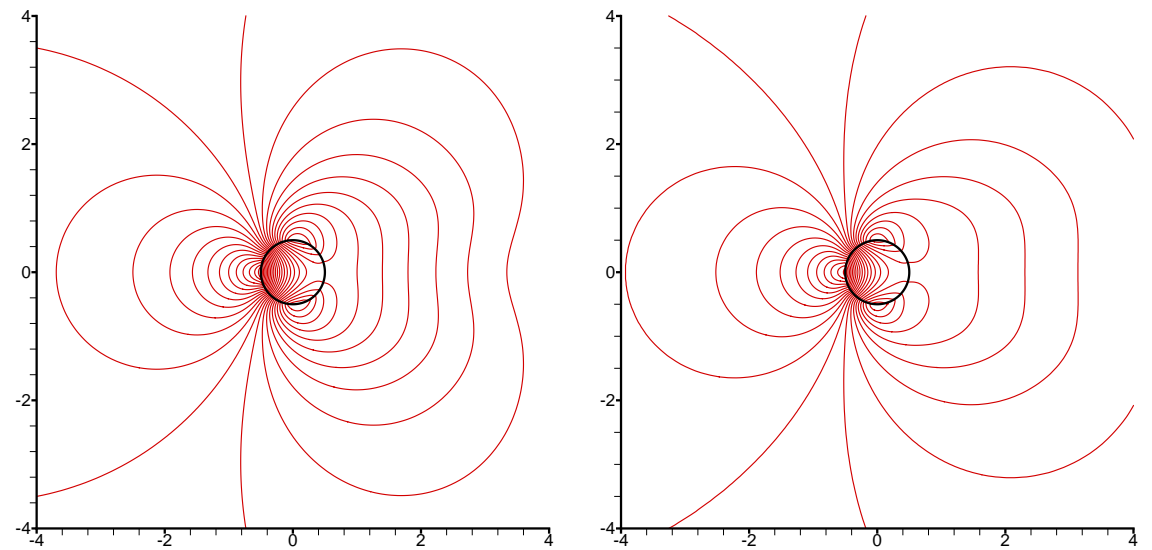

Figure 14: Pressure contours in the flow filed (Left: $R e=20$; Right: $R e=40$ ). 
Strouhal number defined as $S_{t}=f \cdot D / U$ is used to measure the oscillation frequency in the wake region and apparently the current result of $S_{t}=0.167$ is in a good agreement with those from the cited works. The instantaneous flow patterns for a full cycle are shown in Figure 15. The left column displays the streamlines, and the right column exhibits the vorticity contours. The vortex streets formed behind the cylinder are clearly shown in this figure.

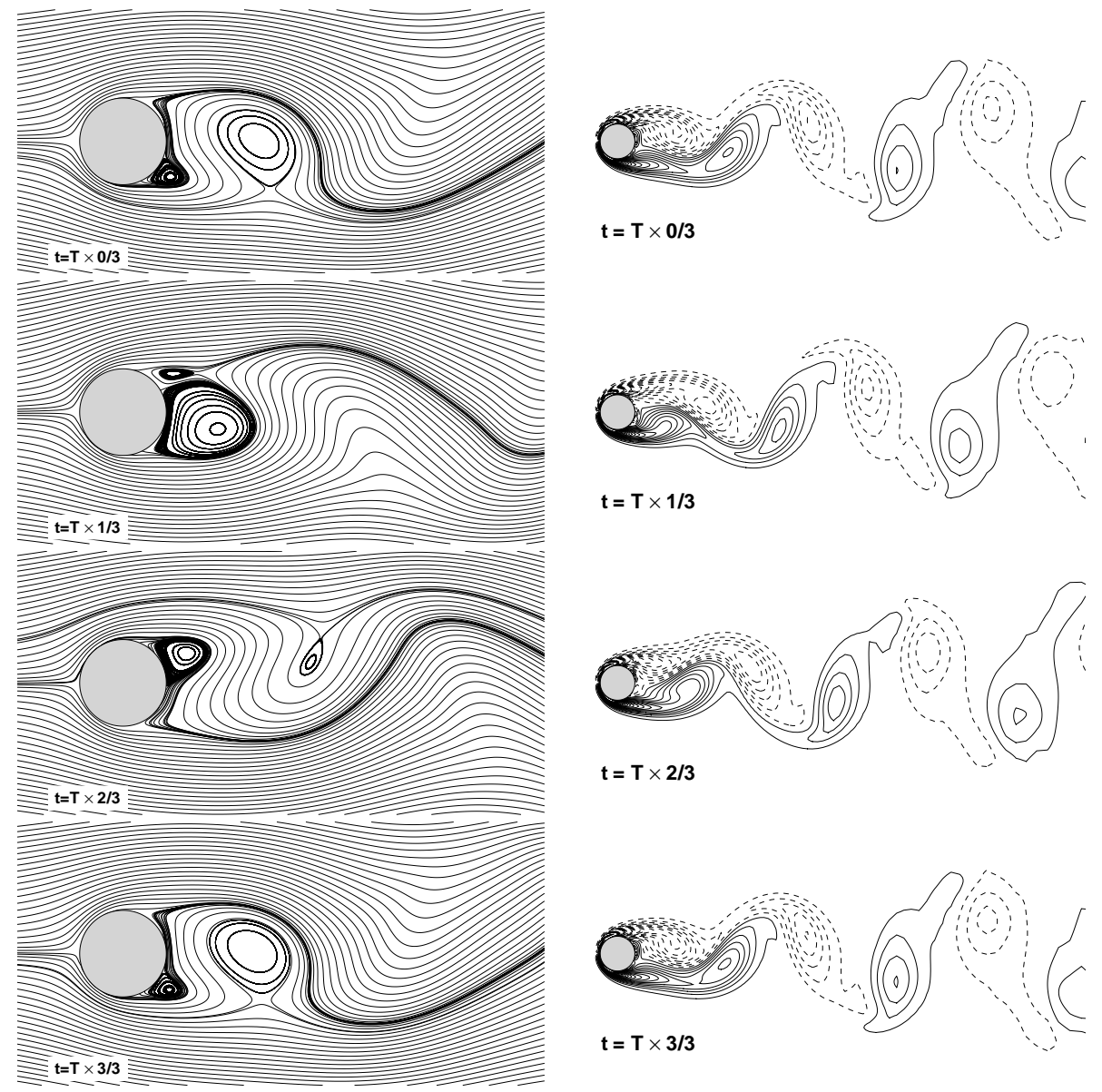

Figure 15: Instantaneous flow patterns in a full cycle for $R e=100$ (Left: streamline; Right: vorticity).

\subsection{Flow past a rotating cylinder}

Incompressible viscous flow past a rotating cylinder has been the subject of theoretical and experimental study for the last several decades due to its importance in the application area of flow control $[15,16]$. Although the center position of 
the cylinder is fixed, this problem is more complicated than flow past a stationary cylinder, as the tangential velocity at the cylinder surface is no longer zero. In this study, it has therefore been chosen to test the capability of the proposed method to deal with flow problems with non-stationary boundaries.

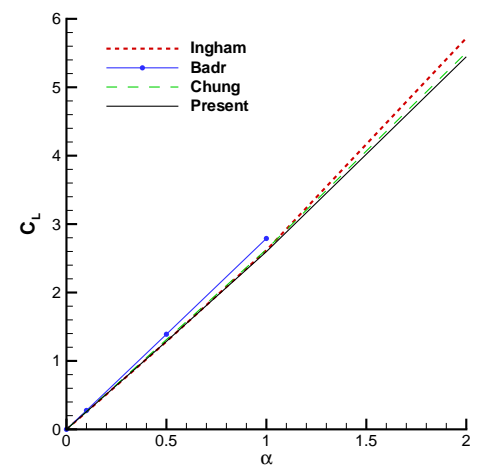

Figure 16: Comparison of lift coefficients $(R e=20)$.

Table 2: Lift and drag coefficients of flows past a rotating cylinder

\begin{tabular}{l|llll|llll}
\hline Contribution & \multicolumn{3}{|c|}{$C_{L}$} & \multicolumn{5}{c}{$C_{D}$} \\
& $\alpha=0.1$ & $\alpha=0.5$ & $\alpha=1$ & $\alpha=2$ & $\alpha=0.1$ & $\alpha=0.5$ & $\alpha=1$ & $\alpha=2$ \\
\hline Ingham et al. [15] & 0.254 & 1.283 & 2.617 & 5.719 & 1.995 & 1.973 & 1.925 & 1.627 \\
Badr et al. [16] & 0.276 & 1.390 & 2.790 & - & 1.990 & 1.970 & 1.910 & - \\
Chung [24] & 0.258 & - & 2.629 & 5.507 & 2.043 & - & 1.888 & 1.361 \\
Present & 0.257 & 1.286 & 2.597 & 5.446 & 1.990 & 1.938 & 1.801 & 1.259 \\
\hline
\end{tabular}

The Reynolds number for the selected test case is 20 and the other dimensionless parameter $\alpha$ for measuring the rotation speed will be used and is defined as

$$
\alpha=\frac{\omega_{o} D}{2 U}
$$

where $\omega_{o}$ is the angular velocity of the rotating cylinder, $D$ is the diameter and $U$ is the free stream velocity. Four test cases with a different rotation speed from $\alpha=0.1$ to $\alpha=2$ are calculated. The corresponding lift coefficients are depicted in Figure 16. The dashed red line is the result of Ingham [15], the solid blue line with symbol represents the work of Badr [16], the dashed green line is the result of Chung [24] and the solid black line is from the present work. It is clearly shown that the lift coefficient $C_{L}$ changes almost linearly with the value of $\alpha$. A detailed comparison of $C_{L}$ and $C_{D}$ between the present work and 

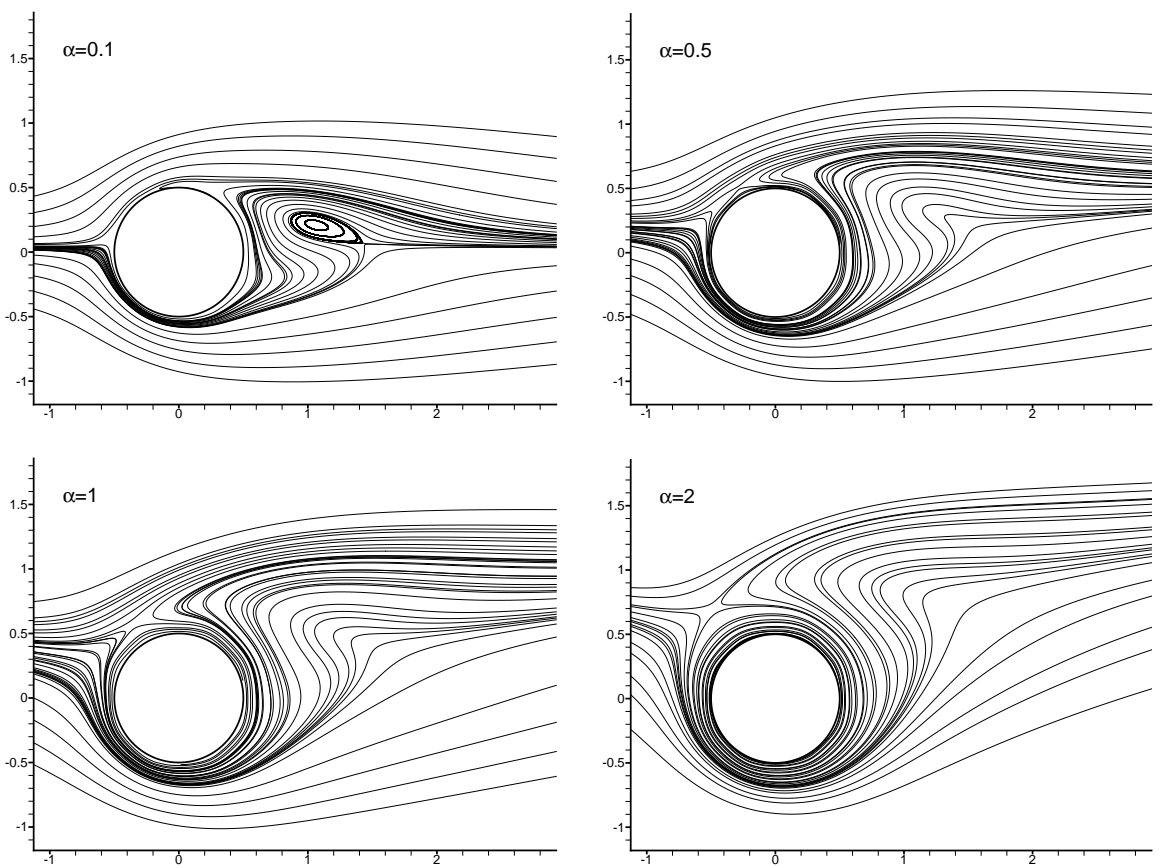

Figure 17: Streamline of flows past a rotating cylinder $(R e=20)$. 
other reference results are given in Table 2. The streamline patterns shown in Figure 17 also agree well with Chung's results [24] (not shown here).

\section{CONCLUSIONS}

In this paper, an efficient Cartesian ghost-cell multigrid Poisson solver is firstly presented. This is then combined with a central finite difference scheme to solve the incompressible Navier-Stokes equations on a rectangular staggered grid using the projection method. With the aid of the ghost cells and their image cells, the boundary conditions at the solid surfaces can be implemented in a straightforward manner. This greatly simplifies the process of both grid generation and the implementation of high order difference schemes. The accuracy and efficiency of the method have been demonstrated initially for the ideal flow past a circular cylinder and then for some test cases of the incompressible viscous flows such as lid driven cavity flow and the flow around both a stationary and a rotating circular cylinder. Future work will include the extension of the method for the solution of the flow problems involving more complex geometries and arbitrary movements.

\section{References}

[1] A.J. Chorin. Numerical solution of the Navier-Stokes Equations. Math. Comp. 1968; 2:745-762.

[2] A.J. Chorin. A numerical method for solving incompressible viscous flow problems. Journal of Computational Physics 1997; 2:12-26.

[3] Bengt Fornberg. A numerical study of steady viscous flow past a circular cylinder. J Fluid Mech 1980; 98:819-855.

[4] Wen-Zhong Shen and Ta-Phuoc Loc. A coupling finite difference/particle method for the resolution of 2D Navier-Stokes equations in velocity-vorticity form. Aerospace Science and Technology 1997; 2:97-109.

[5] G.X. Wu and Z.Z. Hu. Numerical simulation of viscous flow around unrestrained cylinders. Journal of Fluids and Structures 2006; 22:371-390.

[6] X.-Y. Lu and C. Dalton. Calculation of the timing of vortex formation from an oscillating cylinder. Journal of Fluids and Structures 1996; 10:527-541.

[7] Hong-quan Zhang, Uwe Fey and Bernd R. Noack. On the transition of the cylinder wake. Physics of Fluids 1995; 7:779-794.

[8] S. Mittal and V. Kumar. Finte element study of vortex-induced cross-flow and in-line oscillations of a circular cylinder at low reynolds numbers. Int $J$ Numer Meth Fluids 1999; 31:1087-1120. 
[9] H.M. Blackburn and R.D. Henderson. A study of two-dimensional flow past an oscillating cylinder. J Fluid Mech 1999; 385:255-286.

[10] Salvador Pinol and Frances X. Grau Influence of the no-slip boundary condition on the prediction of drag, lift and heat transfer coefficients in the flow past a 2-D cylinder. Numerical Heat Transfer, Part A 1998; 34:313-330.

[11] J.-L. Guermond and L. Quartapelle. On stability and convergence of projection methods based on pressure Poisson equation. Int $J$ Numer Meth Fluids 1998; 26:1039-1053.

[12] R. Webster. Algebraic multigrid solver for Navier-Stokes problems. Int J Numer Meth Fluids 1994; 18:761.

[13] Ghia U, Ghia KN, and Shin CT. High-Re solutions for incompressible flow using the Navier-Stokes equation and a multigrid method. Journal of Computational Physics 1982; 48:387-411.

[14] Tritton D.J. Experiments on the flow past a circular cylinder at low Reynolds number. J Fluid Mech 1959; 6:547.

[15] D.B. Ingham and T. Tang. A numerical investigation into the steady flow past a rotating circular cylinder at low and intermediate Reynods Numbers. Journal of Computational Physics 1990; 87:91-107.

[16] H.M. Bard, S.C.R. Dennis and P.J.S Young. Steady and unsteady flow past a rotating circular cylinder at low Reynolds numbers. Computers 83 Fluids 1989; 17:579-609.

[17] D.J. Kirshman and F. Liu. A gridless boundary condition method for the solution of the Euler equations on embedded Cartesian meshes with multigrid. Journal of Computational Physics 2004; 201:119-147.

[18] Hong Luo, Joseph D. Baum and Rainald Lohner. A hybrid Cartesian grid and gridless method for compressible flows. Journal of Computational Physics 2006; 214:618-632.

[19] C.S. Chew, K.S. Yeo and C. Shu. A generalized finite-difference (GFD) ALE scheme for incompressible flows around moving solid bodies on hybrid meshfree-Cartesian grids. Journal of Computational Physics 2006; 218:510548.

[20] S.J. Ang, K.S. Yeo, C.S. Chew and C. Shu. A singular-value decomposition (SVD)-based generalized finite difference (GFD) method for close-interactin moving boundary flow problems. Int J Numer Meth Engng 2008; 76:18921929.

[21] D.K. Clarke, M.D. Salas, H.A. Hassan. Euler calculations for multielement airfoils uing Cartesian grids. AIAA Journal 1986; 24:353-358. 
[22] D.M. Causon, D.M. Ingram and C.G. Mingham et al. Calculation of shallow water flows using a Cartesian cut cell approach. Advances in Water Resources 2000; 23:545-562.

[23] D.M. Causon, D.M. Ingram and C.G. Mingham et al. A Cartesian cut cell method for shallow water flows with moving boundaries. Advances in Water Resources 2001; 24:899-911.

[24] Meng-Hsuan Chung. Cartesian cut cell approach for simulating incompressible flows with rigid bodies of arbitrary shape. Computers 83 Fluids 2006; 35:607-623.

[25] Hua Ji, Fue-Sang Lien and Eugene Yee. A robust and efficient hybrid cutcell/ghost-cell method with adaptive mesh refinement for moving boundaries on irregular domains. Comput Methods Appl Mech Engrg 2008; 198:432-448.

[26] Daniel Hartmann, Matthias Meinke, Wolfgang Schroder. An adaptive multilevel multigrid formulation for Cartestian hierarchica grid methods. Computers $\&$ Fluids 2008; 37:1103-1125.

[27] Tong Gao, Yu-Heng Tseng and Xi-Yun Lu. An improved hybrid Cartesian/immersed boundary method for fluid-solid flows. International Journal for Numerical Methods in Engineering 2007; 55:1189-1211.

[28] Linwei Shen, Eng-soon Chan, Pengzhi Lin. Calculation of hydrodynamic forces acting on a submerged moving object using immersed boundary method. Computers \& Fluids 2009; 38:691-702.

[29] Yu-Heng Tseng and Joel H. Ferziger. A ghost-cell immersed boundary method for flow in complex geometry. Journal of Computational Physics 2003; 192:593-623.

[30] H. Ding, C. Shu and Q.D. Cai. Applications of stencil-adaptive finite difference method to incompressible viscous flows with curved boundary. Computers $\&$ Fluids 2007; 36:786-793.

[31] Paul Macklin and John S. Lowengrub. A new ghost cell/level set method for moving boundary problems: application to tumor growth. J Sci Comput 2008; 35:266-299.

[32] L.A. Catalano, A. Dadone, V.S.E Daloiso and D. Scardigno. A multigrid procedure for Cartesian ghost-cell methods. Int J Numer Meth Fluids 2008; 58:743-750.

[33] A. Dadone and B. Grossman. Ghost-cell method for inviscid twodimensional flows on Cartesian-grids. AIAA Journal 2004; 42:2499-2507.

[34] A. Dadone and B. Grossman. Ghost-cell method for analysis of inviscid three-dimensional flows on Cartesian-grids. Computers \& Fluids 2007; 36:1513-1528. 
[35] W. Hackbush. Multigrid Methods and Applications. Springer: Berlin, 1985.

[36] R. Barrett, M. Berry and T. F. Chan et al. Templates for the Solution of Linear Systems: Building Blocks for Iterative Methods(2nd Edition). SIAM: Philadelphia, 1994.

[37] J.H. Ferziger and M. Peric. Computational Methods for Fluid(2nd Edition). Springer: Berlin, 2002.

[38] William L. Briggs, Van Emden Henson, Steve F. McCormick. A Multigrid Tutorial(2nd Edition). SIAM: 2000.

[39] P. Wesseling. An introduction to Multigrid Methods. WILEY: New York, 2000.

[40] Pijush K. Kundu, Ira M. Cohen. Fluid Mechanics(3rd Edition). ELSEVIER: 2004. 